\title{
Analysis of Wireless Energy Harvesting Relay Throughput in Rician Channel
}

\author{
Yifan Hu, ${ }^{1}$ Ning Cao, ${ }^{1}$ and Yunfei Chen ${ }^{2}$ \\ ${ }^{1}$ School of Computer and Information, Hohai University, Xikang Road 1, Nanjing 210098, China \\ ${ }^{2}$ School of Engineering, University of Warwick, Coventry CV4 7AL, UK \\ Correspondence should be addressed to Yifan Hu; hhu_huyifan@yeah.net
}

Received 14 June 2016; Revised 10 October 2016; Accepted 23 October 2016

Academic Editor: Francesco Gringoli

Copyright (c) 2016 Yifan Hu et al. This is an open access article distributed under the Creative Commons Attribution License, which permits unrestricted use, distribution, and reproduction in any medium, provided the original work is properly cited.

Cooperative communication uses idle nodes to achieve performance gains. Energy harvesting allows cooperative communication to be less dependent on batteries. In this paper, the performance of energy harvesting (EH) amplify-and-forward relaying is analyzed for Rician fading channels, in contrast to previous works that focused on Rayleigh fading channels. Continuous time EH protocol and discrete time EH protocol are considered. Analytical expressions for the average throughput are derived. Numerical results are presented to show the good performance of the system in Rician fading channels by examining various system parameters using the analytical expressions.

\section{Introduction}

Amplify-and-forward (AF) relaying is widely used in communication systems to extend network coverage or achieve diversity gain. In AF relaying, the information from the source node is received and amplified by the relay node before it is forwarded to the destination node. On the other hand, wireless energy harvesting has an important application in relaying. The relay node may be limited by its battery life so that it may count on some external power source to remain active in the network. In this case, energy harvesting is particularly useful, as the source node can act as an external power source for the relay node by transmitting energy wirelessly.

Several works have been done on either energy harvesting technique or amplify-and-forward relaying technique, to name a few. References [1-3] considered the use of energy harvesting from ambient radio frequency signals to power a wireless sensor network. References $[4,5]$ studied the transmission strategies for $\mathrm{EH}$ nodes. In these cases the nodes harvest energy at some time instant and transmit the harvested energy at some other time instants. In [4], the transmission time minimization was considered. Reference [5] studied the maximized short-time throughput for EH systems.
Reference [6] also studied ambient RF energy harvesting, but instead of using the harvested energy to power the wireless sensor network directly, it considered the option of storing the harvested energy in a capacitor or battery to power the wireless sensor network. In [7], a radio frequency energy harvesting model was analyzed by considering a receiving antenna part, a rectifying part, and an energy storage in the circuit. In [8], the optimum sampling of a random field was studied. Reference [9] studied the best-effort sensing with finite battery. The optimum estimation of a continuous time random process using discrete time samples taken by a sensor with wireless power was studied in [10]. This work showed that, for the sensing of a time-varying random event with wireless power, the optimum performance is achieved when the energy-harvesting-consumption-ratio is 1 . In other studies, the optimal placement of the relay node was also studied. For example, [11] considered a dual-hop energy transfer scheme, while [12] presented a novel optimization problem for two-hop RF energy transfer to improve wireless energy transfer efficiency. In [13], artificial noise was added to reduce the information leakage rate for energy receivers while satisfying its energy harvesting requirements. Reference [14] considered a wireless energy harvesting enabled massive multiple-input-multiple-output relaying system in order to provide wireless security. A protocol using one part of each 
frame for energy harvesting and the other part for message transmission was considered in [15]. In [16], the optimum performance boundaries of a two-hop multiantenna AF relaying system with a multiantenna energy harvesting $(\mathrm{EH})$ receiver were studied. Also, energy harvesting techniques for practical devices in low-power applications were studied using energy harvesting relaying in [17-19]. Reference [20] studied the different rate-energy trade-offs in order to achieve the optimal source and relay coding in a multiple-inputmultiple-output relay system. Reference [21] proposed two protocols of energy harvesting AF relaying and analyzed the throughput of each protocol (continuous time EH protocol and discrete time $\mathrm{EH}$ protocol). All the above works have been conducted for Rayleigh fading channels. However, it is well known that Rayleigh fading channel is only a special case of the Rician fading channel when the line of sight is 0 . Thus, the Rician fading channel is more general and useful than the Rayleigh fading channel, and it is of great interest to investigate the performance $\mathrm{EH}$ relaying in Rician fading channel.

In this paper, we fill this gap by analyzing the performance of energy harvesting AF relaying in Rician fading channels. We consider two EH protocols, similar to [21], as continuous time $\mathrm{EH}$ protocol and discrete time $\mathrm{EH}$ protocol. Based on these protocols, we derive the analytical expression for the throughput in the Rician fading channels, which is more challenging due to the Bessel function in its probability density function. Numerical results are presented to compare the Rician fading channel with the Rayleigh fading channel. They show that the performance in Rician fading channel is better than that in Rayleigh fading channel and this performance gain can be quantified using our results.

The rest of this paper is organized as follows. Section 2 presents the system model of AF relaying system with energy harvesting. In Section 3, we derive the analytical expression for the throughput of each protocol in Rician fading channel. In Section 4, we show the numerical examples obtained from the analytical expressions and verify its accuracy using computer simulation. Section 5 concludes the paper.

\section{System Model}

We consider a cooperative communication scenario constituted by a source node $(\mathbb{S})$, a destination $(\mathbb{D})$, and an intermediate relay node $(\mathbb{R})$. The source node and the destination node are not energy-constrained, but the relay node is energy-constrained. We assume that the destination node can not communicate directly with the source because of physical obstacles. This is a valid assumption in some communication scenarios. Then, deploying EH relay nodes will be a good solution to make the communication between source and destination work effective.

The following assumptions of relaying model are used in this work.

(1) The processing power used for the circuit at the relay is negligible compared with the transmission power required by the relay.
(2) The energy-constrained relay node first harvests sufficient energy from the source and then the relay node relays the source information to the destination using the harvested energy. The battery capacity at the relay is much larger than the required transmission power.

(3) The relay adopts the time-switching (TS) architecture. It spends a portion of time for energy harvesting $(\mathrm{EH})$ and the remaining time for information transmission (IT).

(4) The $\mathbb{S} \rightarrow \mathbb{R}$ and $\mathbb{R} \rightarrow \mathbb{D}$ channels are composed of Rician fading and large-scale path loss. We denote the distances between $\mathbb{S} \rightarrow \mathbb{R}$ and $\mathbb{R} \rightarrow \mathbb{D}$ as $d_{1}$ and $d_{2}$, respectively. The channel fading gains between $\mathbb{S} \rightarrow$ $\mathbb{R}$ and $\mathbb{R} \rightarrow \mathbb{D}$ are denoted, respectively, as $h$ and $g$. The fading channel gains are assumed to be constant over a period of $T$ seconds and are independent and identically distributed from one block to the other.

(5) Assume that the communication happens in blocks of $T$ seconds, each of which is composed of two parts: $\mathrm{EH}$ part and IT part. We denote the fraction of time allocated for $\mathrm{EH}$ in the $i$ th block as $\alpha_{i}$. Thus, EH part takes $\alpha_{i} T$ seconds, $\mathbb{S} \rightarrow \mathbb{R}$ IT part takes $\left(1-\alpha_{i}\right) T / 2$ seconds, and $\mathbb{R} \rightarrow \mathbb{D}$ IT part takes $\left(1-\alpha_{i}\right) T / 2$ seconds. For continuous time $\mathrm{EH}$, the relay harvests energy and transmits information within each block. Thus, $\alpha_{i} \in(0,1)$ [21]. For discrete time EH, the relay uses the whole block for energy harvesting or transmission. When the relay harvests energy, $\alpha_{i}=1$, while when the relay transmits the information, $\alpha_{i}=0$ [21].

(6) For continuous time EH, an EH-IT block contains EH part and IT part, where EH occupies a portion of the block and IT occupies the rest. For discrete time EH, an EH-IT pattern contains $(X+1)$ blocks, where there are $X$ EH blocks and one IT block $(X=0,1,2, \ldots)$.

Also, we define the following symbols:

$E_{0}$ : the available energy at the start of an EH-IT pattern $E_{i}(0)$ : the available energy at the start of the $i$ th block $E_{i}(t)$ : the harvested energy at the time instant $t$ in the $i$ th block

$E_{i}^{0 \rightarrow T}$ : the energy harvested in an $\mathrm{EH}$ block

$t_{0}$ : EH-IT pattern start time

$X$ : the number of $\mathrm{EH}$ blocks of discrete time $\mathrm{EH}$ protocol.

2.1. Signal Model. For the $i$ th block, the source node sends the normalized information signal $s_{i}$ with $\mathbb{E}\left\{\left|s_{i}\right|^{2}\right\}=1$ through the $\mathbb{S} \rightarrow \mathbb{R}$ channel, which is received at the relay node as $y_{r, i}$.

$$
y_{r, i}=\frac{h_{i}}{\sqrt{d_{1}^{m}}} \sqrt{P_{s}} s_{i}+n_{r, i}
$$

where $h_{i}$ is the $\mathbb{S} \rightarrow \mathbb{R}$ fading channel gain, $d_{1}$ is the distance from source to the relay, $m$ is the path loss exponent, $P_{s}$ is 
the source transmission power, and $n_{r, i}$ is the additive white Gaussian noise (AWGN) with mean zero and variance $\sigma_{n r}^{2}$.

Then, the AF relay harvests enough energy to guarantee the required transmission power $P_{r}$ in the IT part. For the general case, $P_{r}$ can be any value set by the requirements of the application QoS, which will produce different throughput. After the EH part, the relay amplifies the received signal and forwards it to the destination node. The signal sent by relay is

$$
x_{r, i}=\frac{\sqrt{P_{r}}}{\sqrt{P_{s}\left|h_{i}\right|^{2} / d_{1}^{m}+\sigma_{n r}^{2}}} y_{r, i} \text {, }
$$

where $P_{s}\left|h_{i}\right|^{2} / d_{1}^{m}+\sigma_{n r}^{2}$ is the average power of $y_{r, i}$ such that

$$
\mathbb{E}\left\{\left|y_{r, i}\right|^{2}\right\}=\frac{P_{s}\left|h_{i}\right|^{2}}{d_{1}^{m}}+\sigma_{n r}^{2}
$$

Finally, the signal $y_{d, i}$ received by the destination node is given by

$$
\begin{aligned}
y_{d, i}= & \frac{g_{i}}{\sqrt{d_{2}^{m}}} x_{r, i}+n_{d, i} \\
= & \frac{\sqrt{P_{r} P_{s}} h_{i} g_{i} s_{i}}{\sqrt{d_{2}^{m}} \sqrt{P_{s}\left|h_{i}\right|^{2}+d_{1}^{m} \sigma_{n r}^{2}}} \\
& +\frac{\sqrt{P_{r} d_{1}^{m}} g_{i} n_{r, i}}{\sqrt{d_{2}^{m}} \sqrt{P_{s}\left|h_{i}\right|^{2}+d_{1}^{m} \sigma_{n r}^{2}}}+n_{d, i} .
\end{aligned}
$$

In (4), $g_{i}$ is the $\mathbb{R} \rightarrow \mathbb{D}$ fading channel gain, $d_{2}$ is the distance of the $\mathbb{R} \rightarrow \mathbb{D}$ link, and $n_{d, i}$ is the AWGN at the destination node. From (4), we can distinguish the signal part and the noise part, where $\sqrt{P_{r} P_{s}} h_{i} g_{i} s_{i} / \sqrt{d_{2}^{m}} \sqrt{P_{s}\left|h_{i}\right|^{2}+d_{1}^{m} \sigma_{n r}^{2}}$ is the signal part and $\sqrt{P_{r} d_{1}^{m}} g_{i} n_{r, i} / \sqrt{d_{2}^{m}} \sqrt{P_{s}\left|h_{i}\right|^{2}+d_{1}^{m} \sigma_{n r}^{2}}+n_{d, i}$ is the noise part. Thus we can get the signal-to-noise ratio (SNR) at the destination node as

$$
\gamma_{d, i}=\frac{P_{s} P_{r}\left|h_{i}\right|^{2}\left|g_{i}\right|^{2} / d_{2}^{m}\left(P_{s}\left|h_{i}\right|^{2}+d_{1}^{m} \sigma_{n r}^{2}\right)}{\sigma_{n d}^{2}+P_{r}\left|g_{i}\right|^{2} d_{1}^{m} \sigma_{n r}^{2} / d_{2}^{m}\left(P_{s}\left|h_{i}\right|^{2}+d_{1}^{m} \sigma_{n d}^{2}\right)},
$$

where again $\sigma_{n d}^{2}$ is the variance of AWGN at $\mathbb{R}$ node.

For the $i$ th block, if the SNR is less than the threshold SNR $\left(\gamma_{0}\right)$ there will be outage. So we get the outage indicator $I_{0, i}$ as

$$
I_{0, i}=\mathbb{L}\left(\gamma_{d, i}<\gamma_{0}\right),
$$

where $\mathbb{L}(\cdot)$ is an indicator function which is equal to 1 if its argument is true; otherwise it is 0 .

\subsection{EH Relay Protocols}

2.2.1. Continuous Time EH Protocol. In this protocol, the relay harvests the energy just enough to transmit the information signal. The continuous time $\mathrm{EH}$ relay makes sure that the relay can harvest the required amount of energy within each block and transmit it to the destination node. The EH-IT pattern only has one block [21].

As Figure 1 shows, firstly the relay spends $\alpha_{i} T$ seconds on harvesting energy, where $\alpha_{i} \in(0,1)$. Then, in the IT part, half of the time $\left(1-\alpha_{i}\right) T / 2$ is for $\mathbb{S} \rightarrow \mathbb{R}$ transmission and the other half time $\left(1-\alpha_{i}\right) T / 2$ is for $\mathbb{R} \rightarrow \mathbb{D}$ transmission. The energy harvested in the EH time will be consumed in the IT time, so that the accumulated harvested energy is 0 . Thus, $E_{0}=E_{i}(0)=0$.

In this case, the harvested energy during $\alpha_{i} T$ seconds is given by

$$
E_{i}\left(\alpha_{i} T\right)=\frac{\eta P_{s}\left|h_{i}\right|^{2}}{d_{1}^{m}} \alpha_{i} T,
$$

where $0<\eta<1$ is the energy conversion efficiency. The relay needs to forward the source message to the destination node with a transmission power $P_{r}$ for a time of $\left(1-\alpha_{i}\right) T / 2$. We can get the relation as

$$
E_{i}\left(\alpha_{i} T\right)=P_{r} \frac{\left(1-\alpha_{i}\right) T}{2} .
$$

So, combining (7) and (8), we can get

$$
\alpha_{i}=\frac{d_{1}^{m} P_{r}}{2 \eta P_{s}\left|h_{i}\right|^{2}+d_{1}^{m} P_{r}} .
$$

In this paper, throughput means the proportion of the effective communication time to the total time when the relaying transmission succeeds. As $\left(1-\alpha_{i}\right) T / 2$ is the effective communication time within one block of time $T$, the throughput $\tau_{i}$ is calculated as

$$
\tau_{i}=\left(1-I_{0, i}\right) \frac{\left(1-\alpha_{i}\right) T / 2}{T}=\frac{\left(1-I_{0, i}\right)\left(1-\alpha_{i}\right)}{2} .
$$

The throughput in (10) is a function of the channel gains $h_{i}$ and $g_{i}$. They are random variables. Thus, the average throughput is

$$
\tau=\mathbb{E}_{h_{i} g_{i}}\left\{\tau_{i}\right\}=\mathbb{E}_{h_{i} g_{i}}\left\{\frac{\left(1-I_{0, i}\right)\left(1-\alpha_{i}\right)}{2}\right\} .
$$

2.2.2. Discrete Time EH Protocol. In this protocol, each block is used either for $\mathrm{EH}$ or for IT. If $E_{i}(0)<P_{r} T / 2$, the block is used for EH. Otherwise, it is used for IT. Within the IT part, $T / 2$ is used for $\mathbb{S} \rightarrow \mathbb{R}$ transmission, and the rest is for $\mathbb{R} \rightarrow \mathbb{D}$ transmission.

In Figure 2, if $E_{0}<P_{r} T / 2$, there will be $X$ EH blocks, and $\mathrm{EH}$ will continue until $E_{i}(0)>P_{r} T / 2$. Then, the relay starts the IT. However, if $E_{0}>P_{r} T / 2$ at the beginning, there will be no $\mathrm{EH}$ part $(X=0)$, and the relay will start IT immediately. In this case, we can see that the harvested energy may not be completely consumed, so that $E_{0}$ can be bigger than zero [21].

In this case, when a block is used for $\mathrm{EH}$, the harvested energy will be

$$
E_{i}^{0 \rightarrow T}=\eta \frac{P_{s}\left|h_{i}\right|^{2}}{d_{1}^{m}} T
$$




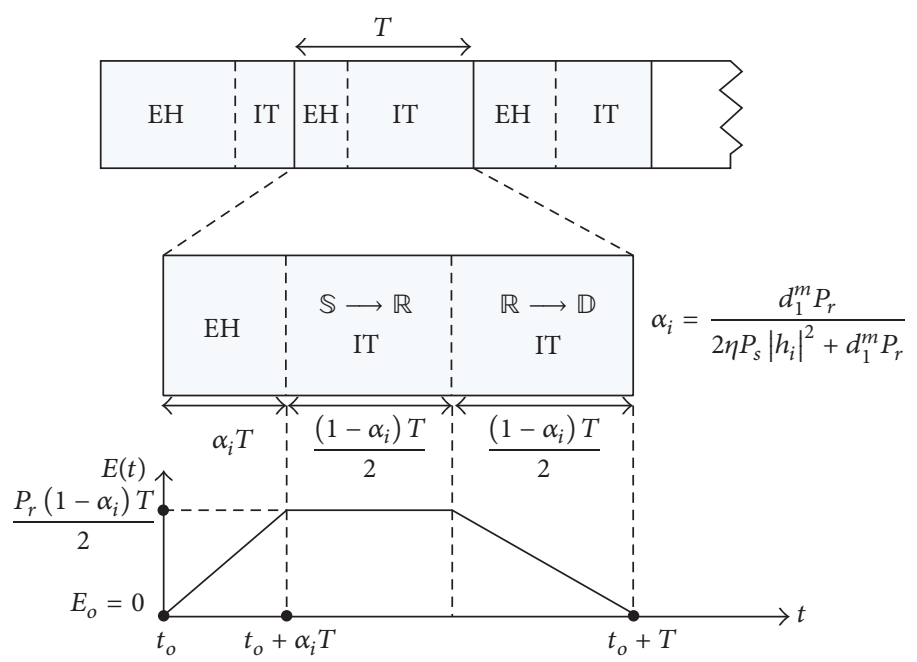

FIGURE 1: The TS protocol for EH and IT in AF relaying with continuous time EH [21].
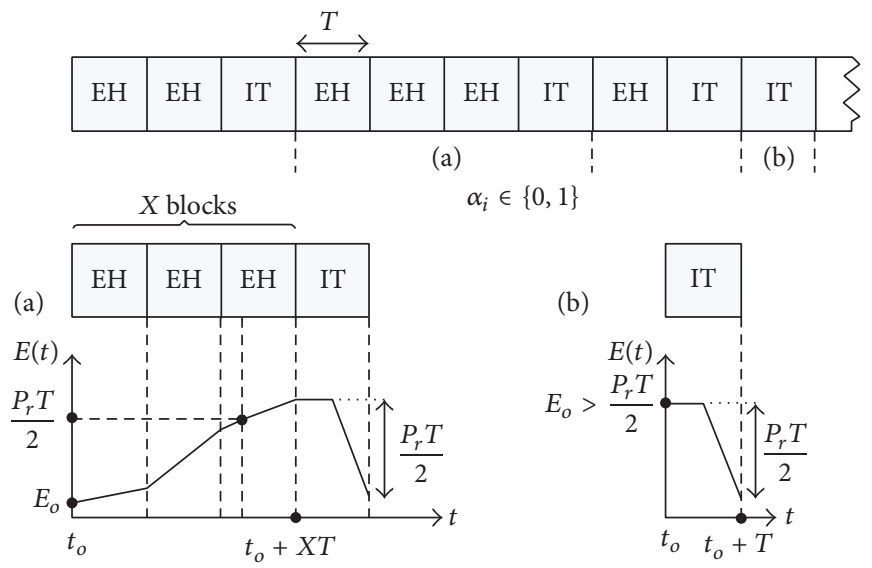

FIGURE 2: The TS protocol for EH and IT in AF relaying with discrete time EH [21].

Then

$$
\begin{gathered}
\alpha_{i}= \begin{cases}1, & E_{i}(0)<\frac{P_{r} T}{2}, \\
0, & E_{i}(0)>\frac{P_{r} T}{2},\end{cases} \\
E_{i}(T)= \begin{cases}E_{i}(0)+E_{i}^{0 \rightarrow T}, & E_{i}(0)<\frac{P_{r} T}{2}, \\
E_{i}(0)-\frac{P_{r} T}{2}, & E_{i}(0)>\frac{P_{r} T}{2} .\end{cases}
\end{gathered}
$$

Similarly, the throughput of the discrete time EH is also $\tau_{i}=\left(1-I_{0, i}\right)\left(1-\alpha_{i}\right) / 2$, and the average throughput is

$$
\tau=\mathbb{E}_{h, g_{i}}\left\{\tau_{i}\right\}
$$

where $h \triangleq\left\{h_{i}, h_{i-1}, h_{i-2}, \ldots\right\}$.

\section{Performance Analysis in Rician Fading Channels}

In this section, we will derive the analytical expression for the throughput of each protocol in Rician fading channels.

3.1. Average Throughput of Continuous Time EH Protocol. Since $h_{i}$ and $g_{i}$ are independent, (11) can be written as

$$
\tau=\mathbb{E}_{h_{i}}\left\{\frac{\mathbb{E}_{g_{i}}\left\{1-I_{0, i}\right\}\left(1-\alpha_{i}\right)}{2}\right\} .
$$

The function of $I_{0, i}$ is given by

$$
\begin{aligned}
I_{0, i} & =\mathbb{L}\left(\frac{P_{s} P_{r}\left|h_{i}\right|^{2}\left|g_{i}\right|^{2}}{P_{r}\left|g_{i}\right|^{2} d_{1}^{m} \sigma_{n r}^{2}+d_{2}^{m} \sigma_{n d}^{2}\left(P_{s}\left|h_{i}\right|^{2}+d_{1}^{m} \sigma_{n r}^{2}\right)}\right. \\
& \left.<\gamma_{0}\right) .
\end{aligned}
$$


For convenience, we define $a=P_{s} d_{2}^{m} \sigma_{n d}^{2} \gamma_{0}, b=$ $d_{2}^{m} \sigma_{n d}^{2} d_{1}^{m} \sigma_{n r}^{2} \gamma_{0}, c=P_{s} P_{r}$, and $d=P_{r} d_{1}^{m} \sigma_{n r}^{2} \gamma_{0}$. Then we have

$$
I_{0, i}= \begin{cases}\mathbb{}\left(\left|g_{i}\right|^{2}<\frac{a\left|h_{i}\right|^{2}+b}{c\left|h_{i}\right|^{2}-d}\right), & \left|h_{i}\right|^{2}>\frac{d}{c}, \\ \mathbb{L}\left(\left|g_{i}\right|^{2}>\frac{a\left|h_{i}\right|^{2}+b}{c\left|h_{i}\right|^{2}-d}\right)=1, & \left|h_{i}\right|^{2}<\frac{d}{c} .\end{cases}
$$

We assume the channel gain is Rician distributed. Thus, the PDF of $\left|h_{i}\right|$ or $\left|g_{i}\right|$ is given by

$$
p_{\left|h_{i}\right|}(x)=\frac{x}{\sigma^{2}} e^{-\left(x^{2}+v^{2}\right) / 2 \sigma^{2}} I_{0}\left(\frac{v x}{\sigma^{2}}\right), \quad x>0,
$$

where $\sigma^{2}$ is the power of the cluttery component and $v$ is the amplitude of the line-of-sight component. Using the transformation $z=x^{2},\left|h_{i}\right|^{2}$ or $\left|g_{i}\right|^{2}$ has the PDF of

$$
p_{\left|h_{i}\right|^{2}}(z)=\frac{1}{2 \sigma^{2}} e^{-v^{2} / 2 \sigma^{2}} e^{-z / 2 \sigma^{2}} I_{0}\left(\frac{v}{\sigma^{2}} \sqrt{z}\right), \quad z>0 .
$$

Then, we get that $\mathbb{E}_{g_{i}}\left\{1-I_{0, i}\right\}$ is

$$
\begin{aligned}
& \mathbb{E}_{g_{i}}\left\{1-I_{0, i}\right\}=1-\frac{1}{2 \sigma^{2}} \\
& \int_{0}^{\left(a\left|h_{i}\right|^{2}+b\right) /\left(c\left|h_{i}\right|^{2}-d\right)} e^{-v^{2} / 2 \sigma^{2}} e^{-z / 2 \sigma^{2}} I_{0}\left(\frac{v}{\sigma^{2}} \sqrt{z}\right) d z, \\
& \left|h_{i}\right|^{2}>\frac{d}{c} .
\end{aligned}
$$

Here, we can see that when $\left|h_{i}\right|^{2}<d / c, \mathbb{E}\left\{1-I_{0, i}\right\}=0$.

Finally we get the average throughput by taking the PDF of $\left|h_{i}\right|^{2}$ into the formula as

$$
\begin{aligned}
\tau= & \int_{d / c}^{\infty} \frac{1-d_{1}^{m} P_{r} /\left(2 \eta P_{s} z+d_{1}^{m} P_{r}\right)}{2}[1 \\
& \left.-\int_{0}^{(a z+b) /(c z-d)} \frac{1}{2 \sigma^{2}} e^{-v^{2} / 2 \sigma^{2}} e^{-x / 2 \sigma^{2}} I_{0}\left(\frac{v}{\sigma^{2}} \sqrt{x}\right) d x\right] \\
& \cdot \frac{1}{2 \sigma^{2}} e^{-v^{2} / 2 \sigma^{2}} e^{-z / 2 \sigma^{2}} I_{0}\left(\frac{v}{\sigma^{2}} \sqrt{z}\right) d z
\end{aligned}
$$

One sees that this is a two-dimensional nested integral, which is not easy to be computed numerically due to convergence issue. So we should do some simplifications. We notice that, in $(20)$, we can get $(a x+b) /(c x-d)=(a / c)(1+$ $\left.(a d+b c) / c^{2} x\right)$. For $(a d+b c) / c^{2}$, it is $d_{2}^{m} \sigma_{n d}^{2} d_{1}^{m} \sigma_{n r}^{2} \gamma_{0} \gamma_{0}^{2} / P_{r} P_{s}=$ $\gamma_{0}^{2} / \mathrm{SINR}_{n r} \mathrm{SINR}_{n d}$. In general cases, the SINR will not be very small, so $(a d+b c) / c^{2}$ is much smaller than 1 . So (20) can be approximated as

$$
\begin{gathered}
1-\int_{0}^{(a z+b) /(c z-d)} \frac{1}{2 \sigma^{2}} e^{-v^{2} / 2 \sigma^{2}} e^{-z / 2 \sigma^{2}} I_{0}\left(\frac{v}{\sigma^{2}} \sqrt{z}\right) d z \\
\approx 1-\int_{0}^{a / c} \frac{1}{2 \sigma^{2}} e^{-v^{2} / 2 \sigma^{2}} e^{-z / 2 \sigma^{2}} I_{0}\left(\frac{v}{\sigma^{2}} \sqrt{z}\right) d z
\end{gathered}
$$

Also,

$$
\begin{aligned}
& \int_{0}^{a / c} \frac{1}{2 \sigma^{2}} e^{-v^{2} / 2 \sigma^{2}} e^{-z / 2 \sigma^{2}} I_{0}\left(\frac{v}{\sigma^{2}} \sqrt{z}\right) d z \\
& \quad=1-\int_{a / c}^{\infty} \frac{1}{2 \sigma^{2}} e^{-v^{2} / 2 \sigma^{2}} e^{-z / 2 \sigma^{2}} I_{0}\left(\frac{v}{\sigma^{2}} \sqrt{z}\right) d z .
\end{aligned}
$$

On the other hand, for $\left(1 / 2 \sigma^{2}\right) \int_{a / c}^{\infty} e^{-v^{2} / 2 \sigma^{2}} e^{-z / 2 \sigma^{2}} I_{0}((v /$ $\left.\left.\sigma^{2}\right) \sqrt{z}\right) d z$, we use the substitution $x^{2}=z / \sigma^{2}$ to have

$$
\begin{aligned}
& \frac{1}{2 \sigma^{2}} \int_{a / c}^{\infty} e^{-v^{2} / 2 \sigma^{2}} e^{-z / 2 \sigma^{2}} I_{0}\left(\frac{v}{\sigma^{2}} \sqrt{z}\right) d z \\
& =\frac{e^{-v^{2} / 2 \sigma^{2}}}{2 \sigma^{2}} \int_{a / c}^{\infty} e^{-z / 2 \sigma^{2}} I_{0}\left(\frac{v}{\sigma^{2}} \sqrt{z}\right) d z \\
& =\frac{e^{-v^{2} / 2 \sigma^{2}}}{2 \sigma^{2}} \int_{\sqrt{a / c} / \sigma}^{\infty} e^{-x^{2} / 2} I_{0}\left(\frac{v}{\sigma} x\right)\left(\frac{d z}{d x}\right) d x \\
& =\int_{\sqrt{a / c} / \sigma}^{\infty} x e^{-\left(x+(v / \sigma)^{2}\right) / 2} I_{0}\left(\frac{v}{\sigma} x\right) d x,
\end{aligned}
$$

where $Q$ function is defined as

$$
Q_{m}(a, b)=\int_{b}^{\infty} x\left(\frac{x}{a}\right)^{m-1} e^{-\left(x^{2}+a^{2}\right) / 2} I_{m-1}(a x) d x .
$$

Then, (25) can be

$$
\int_{\sqrt{a / c} / \sigma}^{\infty} x e^{-\left(x+(v / \sigma)^{2}\right) / 2} I_{0}\left(\frac{v}{\sigma} x\right) d x=Q_{1}\left(\frac{v}{\sigma}, \frac{\sqrt{a / c}}{\sigma}\right) .
$$

So we get

$$
\mathbb{E}_{g_{i}}\left\{1-I_{0, i}\right\} \approx Q_{1}\left(\frac{v}{\sigma}, \frac{\sqrt{a / c}}{\sigma}\right)
$$

Combining (21) and (27), we get

$$
\begin{aligned}
\tau \approx & Q_{1}\left(\frac{v}{\sigma}, \frac{\sqrt{a / c}}{\sigma}\right) \\
& \cdot \int_{0}^{\infty} \frac{1-\alpha_{i}}{2 \sigma^{2}} e^{-v^{2} / 2 \sigma^{2}} e^{-z / 2 \sigma^{2}} I_{0}\left(\frac{v}{\sigma^{2}} \sqrt{z}\right) d z
\end{aligned}
$$

Then we have

$$
\tau \approx \frac{Q_{1}(v / \sigma, \sqrt{a / c} / \sigma)}{2}\left(1-\frac{d_{1}^{m} P_{r}}{2 \sigma^{2}} u\right)
$$


where $u=\int_{0}^{\infty}\left(e^{-v^{2} / 2 \sigma^{2}} e^{-x / 2 \sigma^{2}} I_{0}\left(\left(v / \sigma^{2}\right) \sqrt{x}\right) /\left(2 \eta P_{s} x+\right.\right.$ $\left.\left.d_{1}^{m} P_{r}\right)\right) d x$.

As we can see (29) is a one-dimensional integral from 0 to $\infty$, and due to the exponential function and the Bessel function, the integrand of this integral converges very quickly.

3.2. Average Throughput of Discrete Time EH Protocol. Since $E_{i}^{0 \rightarrow T}=\left(P_{s}\left|h_{i}\right|^{2} \eta / d_{1}^{m}\right) T$ and $\left|h_{i}\right|^{2}$ has the PDF of (19), one has

$$
P_{E_{i}^{0 \rightarrow T}}(\epsilon)=\frac{1}{2 \sigma^{2} \rho} e^{-v^{2} / 2 \sigma^{2}} e^{-\varepsilon / \rho 2 \sigma^{2}} I_{0}\left(\frac{v}{\sigma^{2}} \sqrt{\frac{\epsilon}{\rho}}\right)
$$

where $\epsilon$ is the dummy variable for the random variable $E_{i}^{0 \rightarrow T}$, $\rho=P_{s} \eta T / d_{1}^{m}$, and the mean of $E_{i}^{0 \rightarrow T}$ is $\left(2 \sigma^{2}+v^{2}\right) \rho$.

In the above, the harvested energy is $\sum_{i=1}^{X} E_{i}^{0 \rightarrow T}$. The leftover energy at the end of the first EH-IT pattern after consuming $P_{r} T / 2$ in the IT part is $\sum_{i=1}^{X} E_{i}^{0 \rightarrow T}-P_{r} T / 2$. Afterwards, for the second EH-IT pattern, if the leftover energy of the first EH-IT pattern is smaller than $P_{r} T / 2$, it will be $E_{0}$ for the second EH-IT pattern, but if it is larger than $P_{r} T / 2$, it is also $E_{0}$ of the second EH-IT pattern and will consume another $P_{r} T / 2$ energy without energy harvesting. This process continues. We can deduce that the leftover energy at the end of any EH-IT pattern, in a sequence of EHIT patterns, can be transformed to the same form as $E_{i}^{0 \rightarrow T}$ which is a function of $\rho, v$, and $\sigma$. Consequently, the harvested energy available at the start of any EH-IT pattern, $E_{0}$, will have the PDF:

$$
p_{E_{0}}(\epsilon)=\frac{1}{2 \sigma^{2} \rho} e^{-v^{2} / 2 \sigma^{2}} e^{-\varepsilon / \rho 2 \sigma^{2}} I_{0}\left(\frac{v}{\sigma^{2}} \sqrt{\frac{\epsilon}{\rho}}\right) .
$$

We have

$$
\tau=\mathbb{E}_{h, g_{i}}\left\{\tau_{i}\right\}=\mathbb{E}_{h, g_{i}}\left\{\frac{\left(1-I_{0, i}\right)\left(1-\alpha_{i}\right)}{2}\right\} .
$$

In this case, $I_{0, i}$ is independent of the $\mathrm{EH}$ time, $\alpha_{i}$, but it depends on the fading channels, $h_{i}$ and $g_{i}$; also $\alpha_{i}$ depends on the accumulated harvested energy at the start of the $i$ th block, $E_{i}(0)$. Thus, $E_{i}(0)$ depends on $\mathbb{S} \rightarrow \mathbb{R}$ fading channels of the previous blocks, or $h \backslash h_{i}=\left\{h_{i-1}, h_{i-2}, \ldots\right\}$. So the throughput $\tau$ can be written as

$$
\tau=\frac{1}{2} \mathbb{E}_{h_{i}, g_{i}}\left\{1-I_{0, i}\right\} \mathbb{E}_{h \backslash h_{i}}\left\{1-\alpha_{i}\right\}
$$

Using the results of (20) and (27), we can get

$$
\mathbb{E}_{h_{i}, g_{i}}\left\{1-I_{0, i}\right\} \approx Q_{1}\left(\frac{v}{\sigma}, \frac{\sqrt{a / c}}{\sigma}\right) .
$$

At the same time, we set $X$ as the number of the total EH blocks. Then we denote $\bar{X}=X-1$ as the number of EH blocks just before the energy arrives $P_{r} T / 2$. If $E_{0}>P_{r} T / 2$, there will be $X=0$. When $E_{0}<P_{r} T / 2$, we have known that the mean of $E_{i}^{0 \rightarrow T}$ is $\left(2 \sigma^{2}+v^{2}\right) \rho$. Then we can get $\mathbb{E}\left\{\bar{X} \mid E_{0}\right\}=\left(P_{r} T / 2-\right.$ $\left.E_{0}\right) /\left(2 \sigma^{2}+v^{2}\right) \rho$.

So we have

$$
\mathbb{E}_{\bar{X}}\left\{X \mid E_{0}\right\}= \begin{cases}0, & E_{0}>\frac{P_{r} T}{2}, \\ \frac{P_{r} T / 2-E_{0}}{\left(2 \sigma^{2}+v^{2}\right) \rho}+1, & E_{0}<\frac{P_{r} T}{2} .\end{cases}
$$

Thus

$$
\begin{aligned}
E_{\bar{X}}\{X\}= & \mathbb{E}_{E_{0}}\left\{E_{\bar{X} \mid E_{0}}\left\{X \mid E_{0}\right\}\right\} \\
= & \int_{0}^{P_{r} T / 2}\left(1+\mathbb{E}_{\bar{X}}\left\{\bar{X} \mid E_{0}\right\}\right) p_{E_{0}}(\epsilon) d \epsilon \\
& +\int_{0}^{P_{r} T / 2} 0 p_{E_{0}}(\epsilon) d \epsilon .
\end{aligned}
$$

So we have

$$
\begin{gathered}
E_{\bar{X}}\{X\}=\int_{0}^{P_{r} T / 2}\left(\frac{1}{\left(\sigma^{2}+v^{2}\right) \rho}\left(\frac{P_{r} T}{2}-\epsilon\right)+1\right) \\
\cdot p_{E_{0}}(\epsilon) d \epsilon+\int_{P_{r} T / 2}^{\infty} 0 p_{E_{0}}(\epsilon) d \epsilon \\
=\int_{0}^{P_{r} T / 2}\left(\frac{1}{\left(\sigma^{2}+v^{2}\right) \rho}\left(\frac{P_{r} T}{2}-\epsilon\right)+1\right) \\
\cdot \frac{1}{2 \sigma^{2} \rho} e^{-v^{2} / 2 \sigma^{2}} e^{-\varepsilon / \rho 2 \sigma^{2}} I_{0}\left(\frac{v}{\sigma^{2}} \sqrt{\frac{\epsilon}{\rho}}\right) d \epsilon .
\end{gathered}
$$

We use $Q$ function considered before again, to get

$$
\begin{aligned}
& E_{\bar{X}}\{X\} \\
&=\left(1+\frac{P_{r} T}{2\left(2 \sigma^{2}+v^{2}\right) \rho}\right)\left(1-Q_{1}\left(\frac{v}{\sigma}, \frac{\sqrt{P_{r} T / 2 \rho}}{\sigma}\right)\right) \\
&-\frac{1}{\left(2 \sigma^{2}+v^{2}\right) \rho} \omega,
\end{aligned}
$$

where $\omega=\int_{0}^{P_{r} T / 2}\left(\epsilon / 2 \sigma^{2} \rho\right) e^{-v^{2} / 2 \sigma^{2}} e^{-\varepsilon / \rho 2 \sigma^{2}} I_{0}\left(\left(v / \sigma^{2}\right) \sqrt{\epsilon / \rho}\right) d \epsilon$.

From the definition of throughput we have

$$
\mathbb{E}_{h \backslash h_{i}}\left\{1-\alpha_{i}\right\}=\frac{1 / 2}{E_{\bar{X}}\{X+1\}} .
$$

Combining (34) and (39), the average throughput is

$$
\tau \approx \frac{Q_{1}(v / \sigma, \sqrt{a / c} / \sigma)}{2\left(\left(1+P_{r} T / 2\left(2 \sigma^{2}+v^{2}\right) \rho\right)\left(1-Q_{1}\left(v / \sigma, \sqrt{P_{r} T / 2 \rho} / \sigma\right)\right)-\left(1 /\left(2 \sigma^{2}+v^{2}\right) \rho\right) \omega+1\right)} .
$$




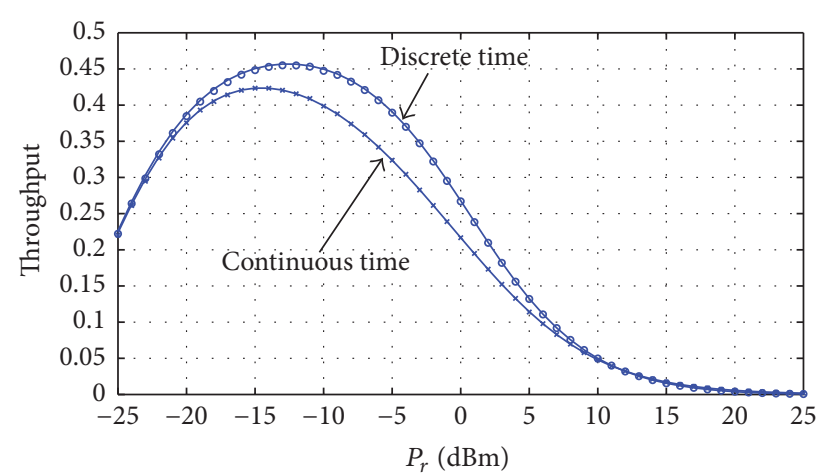

- Analysis of discrete time in Rician fading channel
_ Analysis of continuous time in Rician fading channel
- Simulation of discrete time in Rician fading channel
$\times \quad$ Simulation of continuous time in Rician fading channel

Figure 3: Comparison of the analytical and simulation results for the throughput of discrete time $\mathrm{EH}$ and continuous time $\mathrm{EH}$ in Rician fading channel.

\section{Numerical Result and Discussion}

In this section, numerical results are presented to show the throughput of the system in Rician fading channels. The source transmission power is set to $P_{s}=46 \mathrm{dBm}$. The path loss exponent is set to $m=3$. The distance from source to relay is set to $d_{1}=35$ meters and the distance from relay to destination is set to $d_{2}=10$ meters. The energy conversion efficiency is set to $\eta=0.5$. The threshold SNR is set to $\gamma_{0}=55 \mathrm{~dB}$. The noise variances at the relay and the destination nodes are set to $\sigma_{n r}^{2}=-80 \mathrm{dBm}$ and $\sigma_{n d}^{2}=$ $-110 \mathrm{dBm}$. Also, $\sigma^{2}=0.5$, which is the same as $\sigma$ in the Rayleigh fading channel. We also obtain the simulation results to verify the analytical results obtained in Section 3. In the simulation, 100000 blocks are used, where we generate independent fading channels $h_{i}$ and $g_{i}$ for each block. We assume fixed power transmission at the relay so that $P_{r}$ is fixed while $\alpha_{i}$ changes with $h_{i}$, similar to [21].

Figure 3 shows the comparison of analytical and simulation results for the throughput of discrete time $\mathrm{EH}$ and continuous time EH in Rician fading channel. From Figure 3 we can see that the simulation and analytical result of the throughput of continuous time $\mathrm{EH}$ and discrete time $\mathrm{EH}$ in Rician fading channel match well. This shows the accuracy of the approximation we used. In addition, we can find that there is a peak in the curve. It is because the throughput is related to both the final SNR and the time for $\mathrm{EH}$. When $P_{r}$ increases the final SNR will be larger so that the outage probability will decrease, which makes the throughput larger. On the other side, when $P_{r}$ becomes bigger, the time for $\mathrm{EH}$ will be more, which can decrease the throughput. Therefore, there will be $P_{r}$ that makes the throughput the biggest, which is the optimal. Moreover, we can see that the performance of discrete time $\mathrm{EH}$ protocol is better than that of continuous time EH protocol. There is energy left after a EH-IT pattern of discrete time $\mathrm{EH}$ protocol will harvest more energy when $\left|h_{i}\right|^{2}$ is larger. Then it makes the EH time of the following EHIT pattern smaller, so that the throughput will be larger.

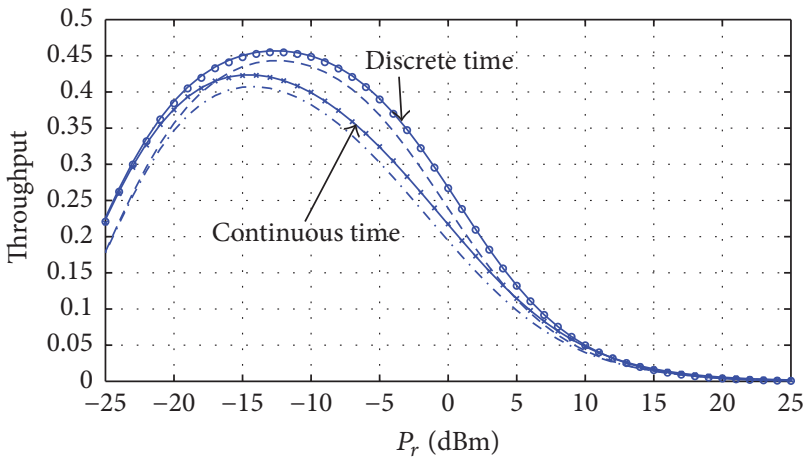

$$
\begin{aligned}
& \text { - Analysis of discrete time in Rician fading channel } \\
& \text { - Analysis of continuous time in Rician fading channel } \\
& \text { - Simulation of discrete time in Rician fading channel } \\
& \text { - Simulation of continuous time in Rician fading channel } \\
& \text { - - - Simulation of discrete time in Rayleigh fading channel }
\end{aligned}
$$

FIgURE 4: Comparison of throughput versus $P_{r}$ for discrete time EH and continuous time EH in Rayleigh and Rician fading channel.

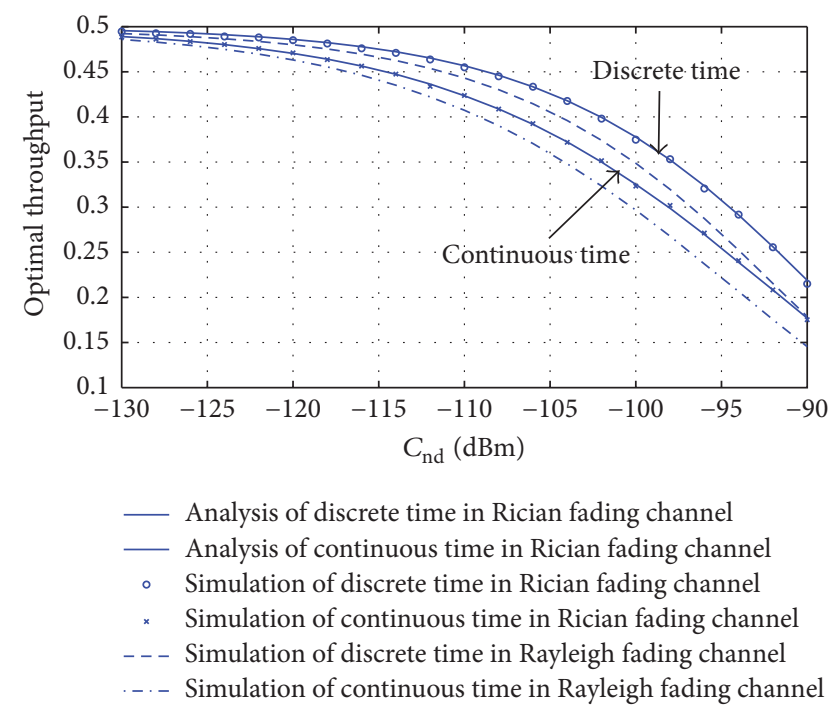

FIGURE 5: Optimal throughput versus $\sigma_{n d}^{2}$ for discrete time EH and continuous time EH in Rayleigh and Rician fading channel.

In Figure 4, the value of $P_{r}$ increases from $-25 \mathrm{dBm}$ to $25 \mathrm{dBm}$. The performance of the throughput of discrete time $\mathrm{EH}$ and continuous time $\mathrm{EH}$ in Rayleigh and Rician fading channels can be compared. From the result, we can see that the throughput in Rician fading channel is higher than that in Rayleigh fading channels under the same other conditions. It conforms to the physical truth that the Rician fading channel is a better fading channel than Rayleigh fading channel, such that performance in Rician fading channel should be better than that in the Rayleigh fading channel.

Figure 5 plots the optimal throughput when the noise variance at the destination nodes increases form $-130 \mathrm{dBm}$ to $-70 \mathrm{dBm}$, so that the throughput also changes when the noise variance changes. The optimum throughput decreases when the noise variance increases. When the noise becomes bigger, $P_{r}$ needs to be bigger to satisfy the outage condition, 


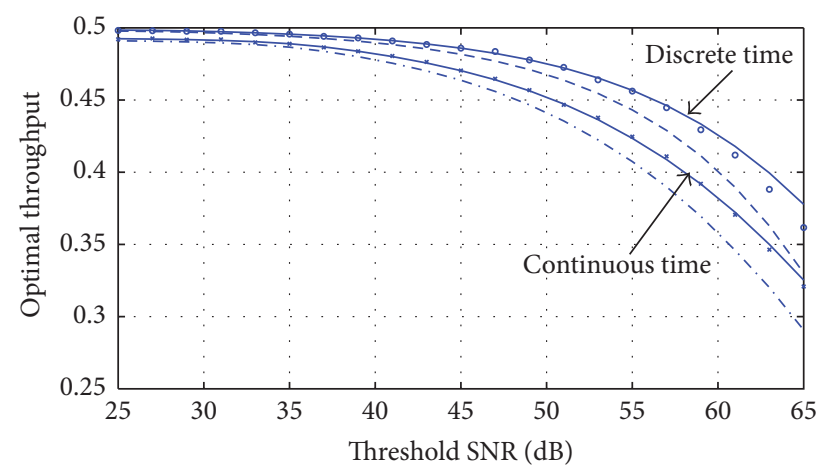

_- Analysis of discrete time in Rician fading channel _ Analysis of continuous time in Rician fading channel

- Simulation of discrete time in Rician fading channel

* Simulation of continuous time in Rician fading channel

- - - Simulation of discrete time in Rayleigh fading channel

-. - Simulation of continuous time in Rayleigh fading channel

FIGURE 6: Optimal throughput versus threshold SNR for discrete time $\mathrm{EH}$ and continuous time $\mathrm{EH}$ in Rayleigh and Rician fading channel.

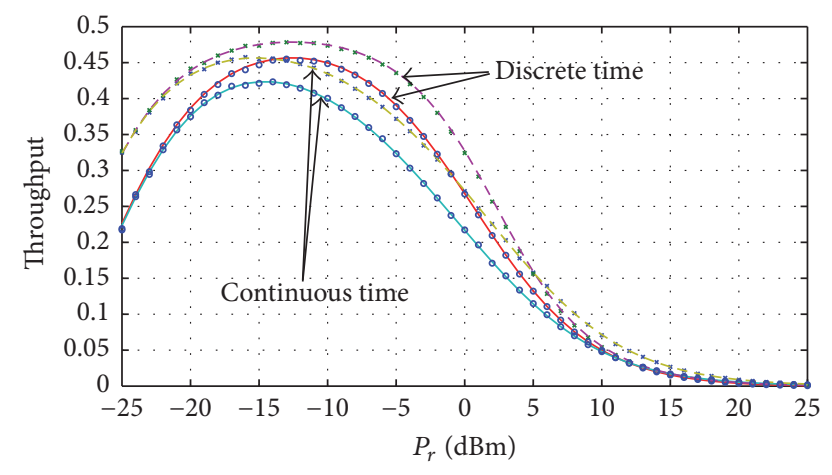

* Rician true discrete time, $v=1$

* Rician true continuous time, $v=1$

Rician discrete time, $v=0.5$

- Rician continuous time, $v=0.5$

- - Rician discrete time, $v=1$

- - Rician continuous time, $v=1$

- Rician true discrete time, $v=0.5$

- Rician true continuous time $v=0.5$

Figure 7: Throughput versus $P_{r}$ for different $v$ in discrete time EH and continuous time $\mathrm{EH}$ in Rician fading channel.

which will make the EH time longer, and finally, makes the throughput decrease.

Figure 6 shows the optimal throughput versus the threshold SNR when it increases from $30 \mathrm{~dB}$ to $70 \mathrm{~dB}$. We can see that when the threshold SNR increases the throughput decreases. The higher threshold SNR needed, the higher final SNR should be, and it will require higher $P_{r}$, which will make the $\mathrm{EH}$ time longer to decrease the throughput. When the threshold SNR increases, the gap between the simulation and analytical results increases, because when the threshold SNR increases the sensibility to $\sigma_{n r}^{2}$ will be bigger, so the approximation becomes inaccurate in this case.

In Figure 7, we examine the line of sight effect. The throughput when $v=1$ is larger than that when $v=0.5$.
When the line of sight increases, that is, the channel becoming better, the throughput will increase.

\section{Conclusion}

In this paper, we have derived the analytical throughput of continuous time and discrete time $\mathrm{EH}$ protocols used in $\mathrm{AF}$ relay in Rician fading channel and have proved the accuracy of the analytical throughput by computer simulation. In addition, we also discussed the performances in different situations for different transmit power of the relaying node, noise at the receive node, and threshold SNR. Moreover, the performance of the EH-IT protocols in Rician fading channel is better than that in Rayleigh fading channel, because of the channel improvement from the line of sight. In addition, the throughput will change while $P_{r}$ changes and there is a peak to attain the optimal throughput, and the optimal throughput will decrease when the noise and threshold SNR increases.

\section{Competing Interests}

The authors declare that they have no competing interests.

\section{References}

[1] A. M. Zungeru, L. M. Ang, S. Prabaharan, and K. P. Seng, "Radio frequency energy harvesting and management for wireless sensor network," in Green Mobile Devices and Networks: Energy Optimization and Scavenging Techniques, pp. 341-368, CRC Press, 2012.

[2] D. Bouchouicha, F. Dupont, M. Latrach, and L. Ventura, "Ambient RF energy harvesting," in Proceedings of the International Conference on Renewable Energies and Power Quality (ICREP '10), Granada, Spain, March 2010.

[3] H. Jabbar, Y. S. Song, and T. T. Jeong, "RF energy harvesting system and circuits for charging of mobile devices," IEEE Transactions on Consumer Electronics, vol. 56, no. 1, pp. 247-253, 2010.

[4] J. Yang and S. Ulukus, "Optimal packet scheduling in an energy harvesting communication system," IEEE Transactions on Communications, vol. 60, no. 1, pp. 220-230, 2012.

[5] K. Tutuncuoglu and A. Yener, "Optimum transmission policies for battery limited energy harvesting nodes," IEEE Transactions on Wireless Communications, vol. 11, no. 3, pp. 1180-1189, 2012.

[6] T. Beng Lim, N. M. Lee, and B. K. Poh, "Feasibility study on ambient RF energy harvesting for wireless sensor network," in Proceedings of the IEEE MTT-S International Microwave Workshop Series on RF and Wireless Technologies for Biomedical and Healthcare Applications (IMWS-BIO '13), pp. 1-3, Singapore, December 2013.

[7] H. ElAnzeery and R. Guindi, "Frequency survey simulation for developing novel radio frequency energy harvesting model," in Proceedings of the 14th International Conference on Modelling and Simulation (UKSim '12), pp. 476-479, March 2012.

[8] K. Ritter, G. W. Wasilkowski, and H. Wozniakowski, "Multivariate integration and approximation for random fields satisfying Sacks-Ylvisaker conditions," The Annals of Applied Probability, vol. 5, no. 2, pp. 518-540, 1995.

[9] J. Yang, X. Wu, and J. Wu, "Adaptive sensing scheduling for energy harvesting sensors with finite battery," in Proceedings of 
the IEEE International Conference on Communications (ICC '15), June 2015.

[10] J. Wu, I. Akingeneye, and J. Yang, "Optimum sensing of a timevarying random event with energy harvesting power sources," in Proceedings of the IEEE International Symposium on Information Theory (ISIT '15), pp. 1134-1138, Hong Kong, June 2015.

[11] A. Kurs, A. Karalis, R. Moffatt, J. D. Joannopoulos, P. Fisher, and M. Soljačić, "Wireless power transfer via strongly coupled magnetic resonances," Science, vol. 317, no. 5834, pp. 83-86, 2007.

[12] D. Mishra and S. De, "Optimal relay placement in two-hop RF energy transfer," IEEE Transactions on Communications, vol. 63, no. 5, pp. 1635-1647, 2015.

[13] L. Liu, R. Zhang, and K.-C. Chua, "Secrecy wireless information and power transfer with MISO beamforming," IEEE Transactions on Signal Processing, vol. 62, no. 7, pp. 1850-1863, 2014.

[14] X. Chen, J. Chen, and T. Liu, "Secure wireless information and power transfer in large-scale MIMO relaying systems with imperfect CSI," https://arxiv.org/abs/1407.5355.

[15] S. Luo, R. Zhang, and T. J. Lim, "Optimal save-then-transmit protocol for energy harvesting wireless transmitters," in Proceedings of the IEEE International Symposium on Information Theory (ISIT '12), pp. 955-959, IEEE, Cambridge, Mass, USA, July 2012.

[16] B. K. Chalise, W.-K. Ma, Y. D. Zhang, H. A. Suraweera, and M. G. Amin, "Optimum performance boundaries of OSTBC based AF-MIMO relay system with energy harvesting receiver," IEEE Transactions on Signal Processing, vol. 61, no. 17, pp. 4199-4213, 2013.

[17] I. Krikidis, "Simultaneous information and energy transfer in large-scale networks with/without relaying," IEEE Transactions on Communications, vol. 62, no. 3, pp. 900-912, 2014.

[18] Y. Zeng and R. Zhang, "Full-duplex wireless-powered relay with self-energy recycling," IEEE Wireless Communications Letters, vol. 4, no. 2, pp. 201-204, 2015.

[19] A. A. Nasir, X. Zhou, S. Durrani, and R. A. Kennedy, "Relaying protocols for wireless energy harvesting and information processing," IEEE Transactions on Wireless Communications, vol.12, no. 7, pp. 3622-3636, 2013.

[20] B. K. Chalise, Y. D. Zhang, and M. G. Amin, "Energy harvesting in an OSTBC based amplify-and-forward MIMO relay system," in Proceedings of the IEEE International Conference on Acoustics, Speech, and Signal Processing (ICASSP '12), pp. 3201-3204, March 2012.

[21] A. A. Nasir, X. Zhou, S. Durrani, and R. A. Kennedy, "Wirelesspowered relays in cooperative communications: time-switching relaying protocols and throughput analysis," IEEE Transactions on Communications, vol. 63, no. 5, pp. 1607-1622, 2015. 

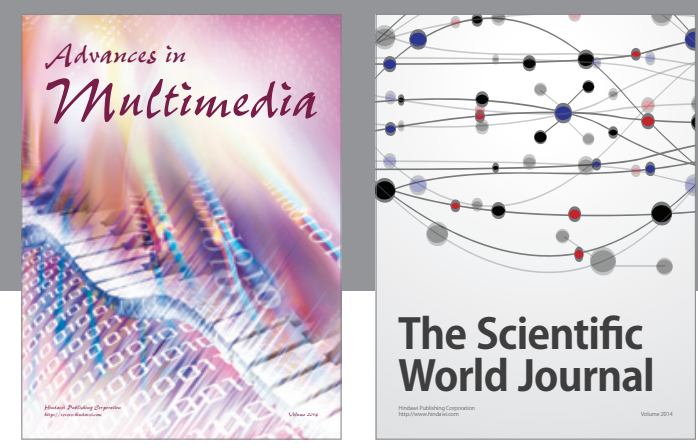

The Scientific World Journal
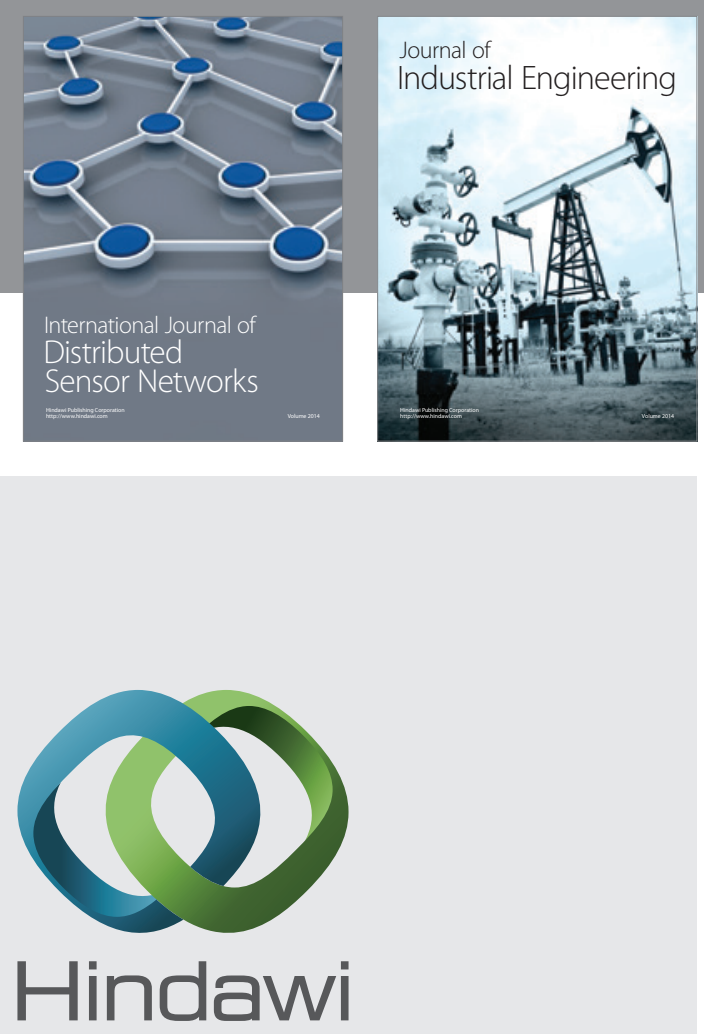

Submit your manuscripts at

http://www.hindawi.com

\section{Computer Networks} and Communications
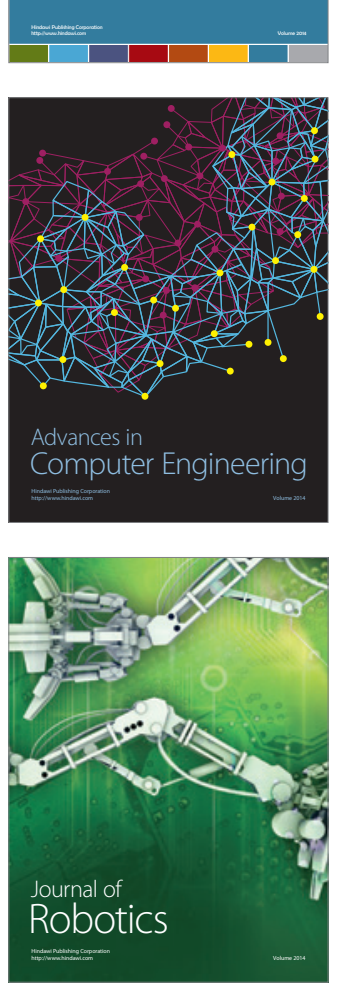
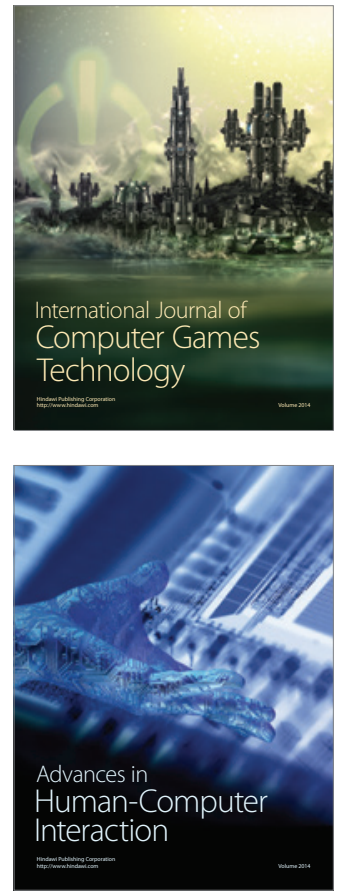
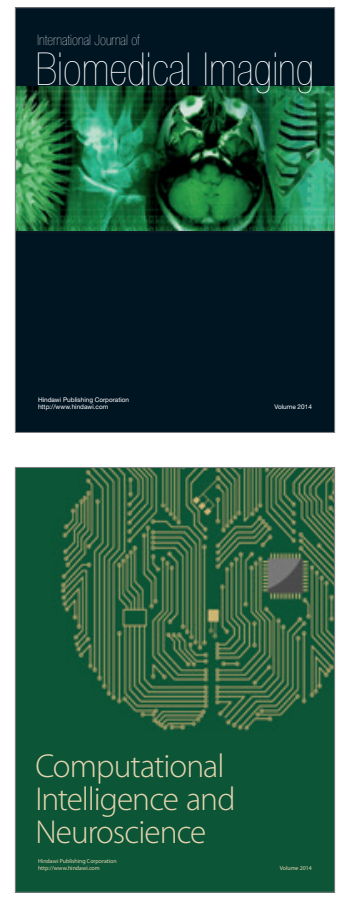
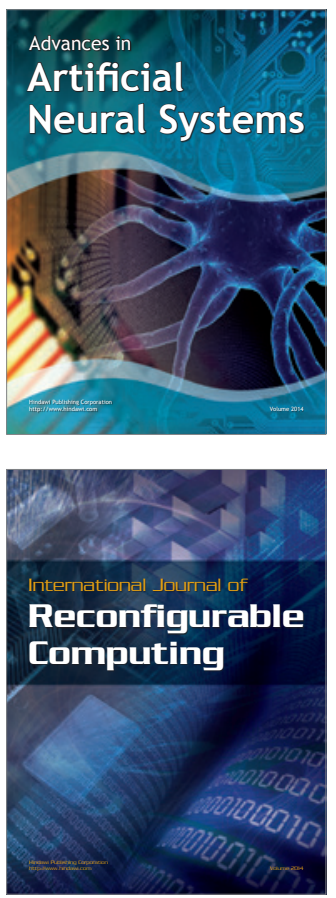
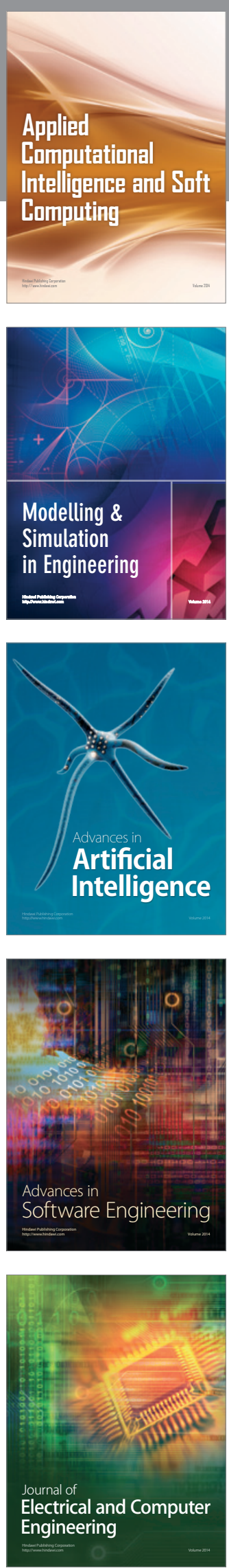